\title{
ESTABILIZAÇÃO QUÍMICA DO SUBLEITO DE ESTRADAS: INFLUÊNCIA DO TEMPO DECORRIDO ENTRE A MISTURA E A COMPACTAÇÃO NA RESISTÊNCIA MECÂNICA DE MISTURAS SOLO-RBI GRADE 81 ${ }^{1}$
}

\author{
Tiago Pinto da Trindade 2 , Dario Cardoso de Lima ${ }^{3}$, Carlos Cardoso Machado ${ }^{4}$, Carlos Alexandre Braz de \\ Carvalho $^{3}$, Carlos Ernesto Gonçalves Reynaud Schaefer ${ }^{5}$, Maurício Paulo Ferreira Fontes ${ }^{5}$ e Fernando \\ Paulo Caneschi ${ }^{6}$
}

\begin{abstract}
RESUMO - Este artigo aborda o estudo da influência do tempo decorrido entre mistura e compactação na resistência mecânica de três solos da Zona da Mata Norte de Minas Gerais, Brasil, quando estabilizados com $4 \%$ de RBI Grade 81, em relação ao peso de solo seco. Um solo residual maduro (solo 1) e dois solos residuais jovens (solos 2 e 3 ) de gnáisse foram utilizados no presente estudo. O programa de ensaios de laboratório englobou: (i) tempos decorridos entre mistura e compactação: 0, 4, 8 e 24 horas; (ii) energia de compactação: Proctor Modificado; (iii) período de cura das misturas: 7 dias; e (iv) determinação da resistência mecânica: média de três determinações da resistência à compressão não-confinada. Os resultados desta pesquisa indicam que: (i) o tempo decorrido entre mistura e compactação dos corpos-de-prova influenciou significativamente o parâmetro resistência à compressão não confinada das misturas; (ii) 4 horas foi o tempo ótimo entre mistura e compactação para as misturas dos solos 1 e 2 com RBI Grade 81; e (iii) no solo 3, foi observado um melhor resultado para a compactação imediatamente após a mistura.
\end{abstract}

Palavras-chave: Estabilização de solos, RBI Grade 81, tempo decorrido entre a mistura e a compactação e resistência à compressão não-confinada.

CHEMICAL STABILIZATION OF ROAD SUBGRADE: INFLUENCE OF ELAPSED TIME BETWEEN MIXTURE AND COMPACTION ON MECHANICAL STRENGTH OF SOIL-RBI GRADE 81 MIXTURES

\begin{abstract}
This paper describes the study of the influence of time between mixture and compaction in the mechanical strength of three soils from "Zona da Mata Norte", Minas Gerais, Brazil, stabilized with 4\% of RBI Grade 81. One mature (soil 1) and two young (soils 2 and 3) gneiss residual soils were used throughout the study. The laboratory testing program consisted of the following steps: (i) elapsed times between mixture and compaction: 0, 4, 8 and 24 hours; (ii) mixture specimen compaction effort: Modified Proctor; (iii) mixture specimen curing time: 7 days in acclimatized room; (iv) determination of mechanical strength: average of
\end{abstract}

\footnotetext{
${ }^{1}$ Recebido em 30.03.2004 e aceito para publicação em 20.04.2005.

${ }^{2}$ Programa de Pós-Graduação em Engenharia Civil da UFV, Viçosa-MG, Brasil. E-mail: <tptrindade@ vicosa.ufv.br>.

${ }^{3}$ Departamento de Engenharia Civil da UFV, Viçosa-MG, Brasil. E-mail: <declima@ufv.br>.

${ }^{4}$ Departamento de Engenharia Florestal da UFV, Viçosa-MG, Brasil. E-mail: <machado@ufv.br>.

${ }^{5}$ Departamento de Solos da UFV, Viçosa-MG, Brasil.

${ }^{6}$ Graduação em Engenharia Civil da UFV, Viçosa-MG, Brasil.
} 
three determinations of unconfined compression strength. The testing data supported that: (i) the elapsed time between mixture and specimen compaction affected significantly the mixture mechanical strength; (ii) 4 hours was the optimum elapsed time for soils 1 and 2 mixtures; (iii) the best result for soil 3 was the compaction effort applied immediately after the mixture.

Key words: Soil stabilization, RBI Grade 81, elapsed time between mixture and compaction, and unconfined compression strength.

\section{INTRODUÇÃO}

O domínio das técnicas de estabilização dos solos pode conduzir a sensíveis reduções nos tempos de execução de obras, viabilizando a industrialização do processo construtivo e, conseqüentemente, propiciando uma economia substancial para o empreendimento (LIMA, 1981; PESSOA, 2004).

As soluções para a estabilização química de solos para fins rodoviários são bastante discutidas na literatura (SILVA, 1968; RICO e DEL CASTILHO, 1977; LIMA, 1981; SENÇO, 2001; LIMA et al., 2003). Mas nos últimos anos, tem havido uma crescente preocupação em buscar estabelecer princípios teóricos que possam explicar os respectivos mecanismos de atuação de cada um dos agentes estabilizantes disponíveis no mercado, particularmente no caso da estabilização química dos solos de clima tropical.

Um aspecto de grande interesse no emprego de aditivos químicos em estradas como agente de cimentação ou aglomerante é a análise da influência do tempo decorrido entre a mistura e a compactação do produto final, haja vista o conjunto de limitações práticas de execução das misturas no campo (SANT'ANA et al., 2003).

Sob esse prisma e com relação ao estabilizante químico RBI Grade 81, o fabricante do produto recomenda, para fins de dosagem das misturas via ensaios de laboratório, que se adote um tempo entre mistura e compactação de 24 horas (ANYWAY, 2003), período esse que difere significativamente daqueles comuns à execução das misturas no campo, em geral inferiores a oito horas. Assim, é de interesse analisar a influência do tempo decorrido entre mistura e compactação sobre a resistência mecânica de misturas solo-RBI Grade 81 . Esse é o tópico abordado no presente artigo, considerando-se os tempos gastos entre mistura e compactação de $0,4,8$ e 24 horas.

\section{MATERIAL E MÉTODOS}

\subsection{Materiais empregados}

Trabalhou-se com um solo residual maduro, pedologicamente classificado como Latossolo VermelhoAmarelo, aqui denominado solo 1, e dois solos residuais jovens, denominados solos 2 e 3 . Esses solos representam solos característicos da Zona da Mata Norte de Minas Gerais, Brasil, em especial da Microrregião de Viçosa.

O solo 1 apresenta evolução pedológica avançada e é proveniente do intemperismo de gnaisse, de ocorrência nas encostas mais suavizadas. Nele predomina a composição mineralógica presente nos argilominerais 1:1 e óxidos de ferro e alumínio. Os solos 2 e 3 são de origem saprolítica, essencialmente quartzosos, com horizonte $\mathrm{C}$ profundo, respectivamente, com textura silto-arenosa a arenosa e coloração rósea a acinzentada. Nos Quadros 1 e 2, encontram-se, respectivamente, informações sobre a distribuição granulométrica, os limites de Atterberg e o peso específico dos grãos, as classificações dos três solos, segundo os Sistemas TRB Transportation Research Board, USC Unified Soil Classification e a Metodologia MCT (Miniatura, Compactado e Tropical), preconizada por Nogami e Villibor (1995).

No que diz respeito à classificação geotécnica MCT e segundo as considerações feitas por Nogami e Villibor (1995), pode-se esperar que os solos 1, 2 e 3 apresentem as seguintes características: (i) solo 1 , os integrantes do grupo LG', quando possuem porcentagem relativamente elevada de grãos de areia e são devidamente compactados, podem apresentar elevada capacidade de suporte, elevado módulo de resiliência, baixa permeabilidade, pequena contração por perda de umidade, razoável coesão e pequena expansibilidade por imersão em água; (ii) solo 2 , os solos do grupo NS' (solos siltosos não-lateríticos) se 
caracterizam principalmente por terem, quando compactados na umidade ótima e peso específico aparente seco máximo da energia normal, baixa capacidade de suporte quando imersos em água, baixo módulo de resiliência, elevada erodibilidade, elevada expansibilidade (porém baixa pressão de expansão), elevado coeficiente de sorção e permeabilidade média; e (iii) solo 3, os solos típicos do grupo NG', quando compactados nas condições de umidade ótima e peso específico aparente seco máximo da energia normal, apresentam características das argilas tradicionais muito plásticas e expansivas. Do ponto de vista da resiliência, apresentam, quando compactados, características bilineares em função da tensão-desvio, sendo pouco dependentes da pressão de confinamento. $\mathrm{O}$ seu emprego se prende às restrições conseqüentes de suas elevadas características de expansibilidade, plasticidade, compressibilidade e contração, quando submetidos à secagem.

O estabilizante químico empregado na presente pesquisa foi o RBI Grade 81. Atualmente, esse aditivo químico é produzido pela empresa Anyway Solid Environmental Solutions LTD, em Israel e no Canadá. Até o final de 2003, possuía representação no Brasil através da companhia TECFLORA S.A., que gentilmente forneceu amostras do produto para a realização deste trabalho.

\subsection{Preparação de amostras e programa de ensaios}

Foram analisadas amostras dos solos no estado natural e quando estabilizados com $4 \%$ de RBI Grade 81 em relação ao peso de solo seco, englobando os ensaios geotécnicos de laboratório descritos nos tópicos subseqüentes.

\subsubsection{Ensaios de compactação}

Os ensaios de compactação foram realizados na energia do ensaio Proctor Modificado, segundo a metodologia descrita na Norma Técnica NBR 7182/86 (ABNT, 1986), para fins de determinação do peso específico aparente seco máximo $\left(\gamma_{\text {dmáx }}\right)$ e da umidade ótima $\left(\mathrm{w}_{\mathrm{ot}}\right)$ em solos no estado natural e após a incorporação do aditivo químico.

\subsubsection{Moldagem dos corpos-de-prova}

Os corpos-de-prova utilizados nos ensaios de compressão não-confinada foram moldados nos parâmetros ótimos ( $\left.\mathrm{w}_{\mathrm{ot}} \mathrm{e} \gamma_{\mathrm{dmáx}}\right)$ da energia de compactação

Quadro 1 - Distribuição granulométrica (ABNT, 1995), limites de Atterberg, peso específico dos sólidos e classificação dos solos segundo os sistemas TRB e USC

Table 1 - Grain size distribution (ABNT, 1995), Atterberg limits, grain unit weight and classification of soils according to the TRB and USC systems

\begin{tabular}{|c|c|c|c|c|}
\hline & \multirow[t]{2}{*}{ Propriedades Analisadas } & \multicolumn{3}{|c|}{ Designação das Amostras } \\
\hline & & Solo 1 & Solo 2 & Solo 3 \\
\hline \multirow{12}{*}{ 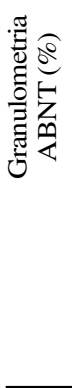 } & Argila $(\phi \leq 0,002 \mathrm{~mm})$ & 61 & 15 & 13 \\
\hline & Silte $(0,002<\phi \leq 0,06 \mathrm{~mm})$ & 14 & 22 & 17 \\
\hline & Areia fina $(0,06<\phi \leq 0,2 \mathrm{~mm})$ & 10 & 30 & 13 \\
\hline & Areia média $(0,2<\phi \leq 0,6 \mathrm{~mm})$ & 12 & 26 & 37 \\
\hline & Areia grossa $(0,6<\phi \leq 2 \mathrm{~mm})$ & 3 & 7 & 20 \\
\hline & Pedregulho $(\phi>2 \mathrm{~mm})$ & 0 & 0 & 0 \\
\hline & $\operatorname{LL}(\%)$ & 74 & 38 & 29 \\
\hline & LP $(\%)$ & 37 & 25 & 20 \\
\hline & IP (\%) & 37 & 13 & 9 \\
\hline & $\gamma_{\mathrm{s}}\left(\mathrm{KN} / \mathrm{m}^{3}\right)$ & 27,05 & 25,44 & 25,24 \\
\hline & Classificação TRB & A-7-5 (20) & A-6 (1) & A-2-4 $(0$ \\
\hline & Classificação USC & MH & SM & SC \\
\hline
\end{tabular}

Quadro 2 - Índices classificatórios e classificação MCT dos solos Table 2 - Classification indexes and MCT classification of soils

\begin{tabular}{|c|c|c|c|c|c|}
\hline Designação das Amostras & Índice c' & Índice d' & $\mathrm{Pi}(\%)$ & Índice e' & Classificação MCT \\
\hline Solo 1 & 1,95 & 103,15 & 0 & 0,58 & LG' $^{\prime}$ \\
\hline Solo 2 & 1,27 & 8,42 & 136 & 1,55 & NS' \\
\hline Solo 3 & 1,22 & 31,28 & 125 & 1,25 & NG' \\
\hline
\end{tabular}


anteriormente citada. Os corpos-de-prova foram compactados por processo dinâmico em cinco camadas iguais, aplicando-se 27 golpes com um soquete de massa igual a 4,536 kg e altura de queda de 45,72 cm em cada camada e utilizando o molde do ensaio de compactação Proctor, ou seja, um molde cilíndrico de $10 \mathrm{~cm}$ de diâmetro e $12,73 \mathrm{~cm}$ de altura (aproximadamente, $1.000 \mathrm{~cm}^{3} \mathrm{de}$ volume). Para aceitação dos corpos-de-prova foram fixados os seguintes limites em sua preparação (DNER, $1994_{\mathrm{b}}$ ): peso específico aparente seco máximo $\pm 0,30$ $\mathrm{kN} / \mathrm{m}^{3}$ e umidade ótima $\pm 0,5 \%$.

\subsubsection{Ensaios de compressão não-confinada}

Os ensaios de compressão não-confinada, dos três solos analisados foram realizados segundo a metodologia proposta pelo Departamento Nacional de Estradas de Rodagem (DNER, 1994 ), para fins de determinação do tempo ótimo entre a mistura e a compactação. Para execução desses ensaios, consideraram-se a energia de compactação do ensaio Proctor Modificado, o período de cura em câmara úmida de sete dias e o teor de RBI Grade 81 de $4 \%$ em relação ao peso de solo seco. A determinação da resistência à compressão não-confinada foi realizada pela média aritmética das tensões de ruptura de três corpos-deprova, admitindo-se uma tolerância de $\pm 10 \%$ em torno da média. Nos casos em que o desvio-padrão superou o valor preestabelecido, procedeu-se à moldagem e execução do ensaio de outros três corpos-de-prova, aumentando, assim, o número de amostras e, conseqüentemente, a confiabilidade dos resultados.

\section{RESULTADOS E DISCUSSÕES}

No Quadro 3, encontram-se os parâmetros ótimos de compactação ( $\mathrm{w}_{\mathrm{ot}}$ e $\gamma_{\text {dmáx }}$ ) determinados para os solos 1,2 e 3, bem como para as respectivas misturas.

A partir dos valores apresentados no Quadro 3, notou-se que a adição de RBI Grade 81 foi responsável por alterações nos parâmetros ótimos de compactação dos solos estudados. A adição de $4 \%$ de estabilizante, em relação ao peso seco de solo, promoveu reduções da ordem de 2,2 e $7 \%$ nos valores de peso específico aparente seco máximo, respectivamente, nos solos 1 , 2 e 3 . Verificaram-se, também, aumentos da ordem de 4, 5 e $21 \%$ nos valores de umidade ótima, respectivamente, nos solos 1, 2 e 3 . Acredita-se que o aumento da umidade ótima com o acréscimo do teor de aditivo químico nas misturas deva-se ao maior consumo da água necessária às reações de hidratação do estabilizante, pois o RBI Grade 81 é considerado um cimento hidráulico. Já as reduções dos valores de peso específico aparente seco máximo podem ser atribuídas à formação de flóculos, estabelecendo-se ligações entre as partículas menores e favorecendo (pelo agrupamento desses agregados menores formados pela ação do aditivo químico) a formação de agregados maiores que, conseqüentemente, produzem um material com estrutura ligeiramente mais porosa.

As Figuras 1, 2 e 3 ilustram, respectivamente, os valores da resistência à compressão não-confinada nos solos 1,2 e 3, bem como a variação desse parâmetro com o tempo decorrido entre a mistura e a compactação das misturas solo-RBI Grade 81.

Analisando os dados apresentados nas Figuras 1 e 2, observou-se que a resistência máxima obtida ocorreu após quatro horas de repouso da mistura, cujo valor foi de $1.330 \mathrm{kPa}$ no solo 1 e $1.341 \mathrm{kPa}$ no solo 2. No período entre a mistura e a compactação de quatro horas, os ganhos de resistência das misturas em relação aos solos 1 e 2 foram, respectivamente, de 52 e $110 \%$. No caso em que as misturas ficaram em repouso por um período de 24 horas, conforme recomendações do fabricante do RBI Grade 81 (ANYWAY, 2003), os ganhos de resistência das misturas em relação aos solos $1 \mathrm{e}$ 2 foram, respectivamente, de 34 e $86 \%$. Esse comportamento pode ser atribuído à ocorrência de reações de trocas catiônicas entre o aditivo e o solo e, consequientemente, o consumo inicial de estabilizante, anteriormente à formação de ligações de cimentação que, provavelmente, têm início depois das primeiras quatro horas após a mistura dos solos 1 e 2 com o aditivo químico.

Quadro 3 - Resultados dos ensaios de compactação Table 3 - Compaction test data

\begin{tabular}{ccccccc}
\hline Porcentagem de RBI Grade 81 & \multicolumn{3}{c}{ Solo 1 } & \multicolumn{2}{c}{ Solo 2 } & \multicolumn{2}{c}{ Solo 3 } \\
\cline { 2 - 7 } & $\gamma_{\text {dmáx }}\left(\mathrm{kN} / \mathrm{m}^{3}\right)$ & $\mathrm{w}_{\mathrm{ot}}(\%)$ & $\gamma_{\mathrm{dmax}}\left(\mathrm{kN} / \mathrm{m}^{3}\right)$ & $\mathrm{w}_{\mathrm{ot}}(\%)$ & $\gamma_{\mathrm{dmax}}\left(\mathrm{kN} / \mathrm{m}^{3}\right)$ & $\mathrm{w}_{\mathrm{ot}}(\%)$ \\
\hline 0 & 15,17 & 27,32 & 17,49 & 14,31 & 19,68 & 9,95 \\
4 & 14,94 & 28,44 & 17,09 & 15,09 & 18,30 & 12,06 \\
\hline
\end{tabular}

R. Árvore, Viçosa-MG, v.29, n.3, p.413-418, 2005 


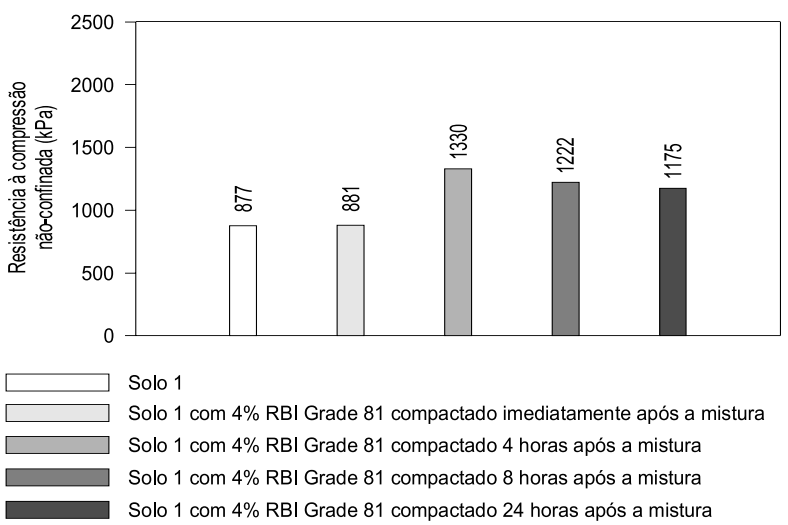

Figura 1 - Resultados dos ensaios de compressão não-confinada: solo 1 .

Figure 1 - Unconfined compression test data: soil 1.

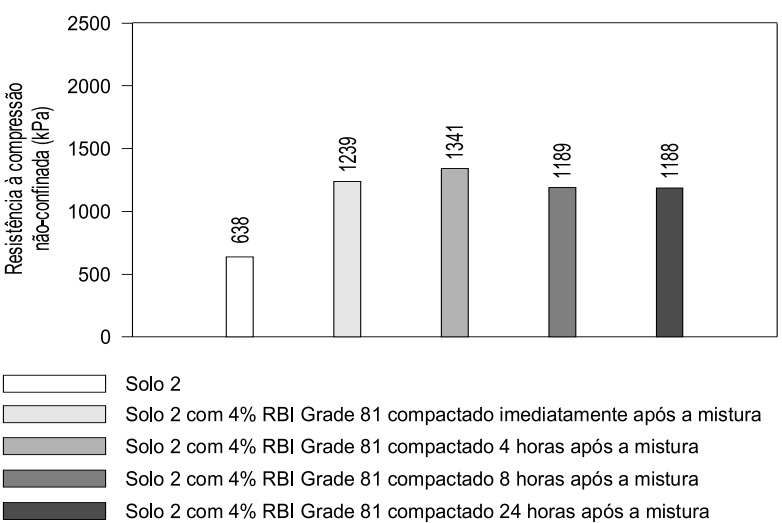

Figura 2 - Resultados dos ensaios de compressão não-confinada: solo 2 .

Figure 2 -Unconfined compression test data: soil 2.

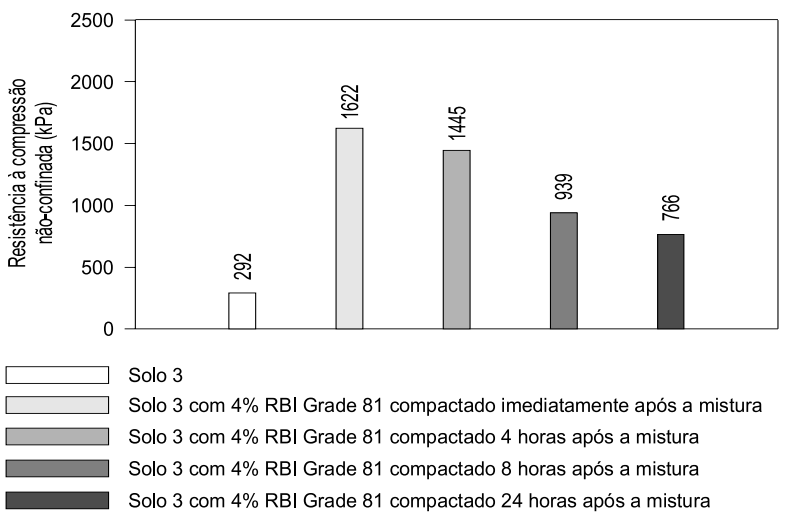

Figura 3 - Resultados dos ensaios de compressão não-confinada: solo 3 .

Figure 3 - Unconfined compression test data: soil 3
Em contrapartida aos resultados discutidos no parágrafo anterior, no solo 3 os dados ilustrados na Figura 3 indicam que a resistência à compressão nãoconfinada máxima ocorreu nos corpos-de-prova compactados imediatamente após a mistura desse solo com o RBI Grade 81, sendo observado um valor de $1.622 \mathrm{kPa}$, o que corresponde a um ganho de resistência de $455 \%$. Quando a mistura do solo 3 ficou em repouso por um período de 24 horas, o ganho de resistência foi da ordem de $162 \%$. Nesse caso, acredita-se que tenha ocorrido a formação de ligações de cimentação imediatamente após a mistura do solo com o aditivo, as quais podem ter sido parcialmente quebradas durante a compactação dos corpos-de-prova, causando, conseqüentemente, reduções na resistência mecânica das misturas. No entanto, devido ao fato de o processo de hidratação ser relativamente rápido nessas misturas estabilizadas, à semelhança de misturas solo-cimento, advoga-se, também, que possam ter ocorrido reações de cimentação mais efetivas no material que foi imediatamente compactado após a mistura.

\section{CONCLUSÕES}

Com base nos resultados, pode-se concluir que o tempo decorrido entre a mistura e a compactação, aspecto fundamental para a boa execução das camadas estabilizadas no campo, é de importância significativa na presente modalidade de estabilização.

A mistura do solo 3 com o aditivo químico RBI Grade 81 foi a que apresentou maior ganho de resistência. Entretanto, essa mesma mistura foi a que teve queda acentuada de resistência relacionada a maiores períodos decorridos entre a mistura e a compactação. Assim, recomenda-se que a execução no campo de camadas do pavimento contendo misturas de RBI Grade $81 \mathrm{com}$ solos granulares (semelhantes ao solo 3 ) não exceda quatro horas.

Os ensaios realizados em laboratório conforme as recomendações do fabricante, ou seja, amostras compactadas após um período de repouso das misturas de 24 horas, subestimaram os valores de resistência à compressão não-confinada e, conseqüentemente, mostraram-se a favor da segurança, uma vez que a execução no campo de camadas do pavimento contendo essas misturas geralmente ocorre num período máximo de oito horas.

R. Árvore, Viçosa-MG, v.29, n.3, p.413-418, 2005 
De maneira geral, as misturas dos solos estabilizados com RBI Grade 81, quando comparadas com os solos no estado natural, apresentaram melhorias nas suas características mecânicas, indicando que o aditivo químico estudado pode ser utilizado, com sucesso, como revestimento primário de estradas de baixo volume de tráfego (florestais e vicinais, por exemplo) e como camadas de pavimentos tradicionais.

\section{AGRADECIMENTOS}

À CAPES, pela concessão da bolsa de estudo ao primeiro autor; à SIF, pelo apoio financeiro; e à Universidade Federal de Viçosa, através do Departamento de Engenharia Civil, por ter possibilitado a execução do presente trabalho.

\section{REFERÊNCIAS BIBLIOGRÁFICAS}

\section{ASSOCIAÇÃO BRASILEIRA DE NORMAS} TÉCNICAS - ABNT. Solo: ensaio de compactação - Procedimento. NBR 7182/ 86. Rio de Janeiro: 1986, 10p.

\section{ASSOCIAÇÃO BRASILEIRA DE NORMAS} TÉCNICAS - ABNT. Rochas e solos. NBR 6502/95. Rio de Janeiro: 1995. 18p.

ANYWAY. Guide to the construction of pavement layers and stabilized materials using RBI Grade 81 natural soil stabilizer. Disponível em http:// www.anywaysolutions.com/. Anyway Solid Environmental Solutions LTD. Kerem Maharal, Israeli Office, 50 Bezalel Street, Ramat-Gan, 52521 Israel, 2003. 8p. Acesso em 22 março de 2005.

\section{DEPARTAMENTO NACIONAL DE ESTRADAS} DE RODAGEM - DNER Solo-cimento: compressão axial de corpos-de-prova cilíndricos. Método de Ensaio DNER-ME 201/ 94. Rio de Janeiro: 1994a. 4p.

\section{DEPARTAMENTO NACIONAL DE ESTRADAS} DE RODAGEM - DNER Solo-cimento: moldagem e cura de corpos-de-prova cilíndricos. Método de Ensaio DNER-ME 202/ 94. Rio de Janeiro: 1994b. 7p.
LIMA, D.C. Algumas considerações relativas a aspectos da estabilização dos solos, em particular à estabilização solo-cal. 1981. 171f. Dissertação (Mestrado em Geotecnia), Escola de Engenharia de São Carlos, São Carlos-SP, 1981.

LIMA, D.C. et al. A experiência da UFV no emprego de estabilizantes químicos em estradas florestais. In: BRAZILIAN SYMPOSIUM ON HARVESTING AND WOOD TRANSPORT, 6. 2003. Proceedings... Viçosa, MG: Sociedade de Investigações Florestais, 2003. p. 69-117.

NOGAMI, J.S.; VILLIBOR, D.F. Pavimentação de baixo custo com solos lateríticos. São Paulo: 1995. 213p.

PESSOA, F.H.C. Análises dos solos de Urucu para fins de uso rodoviário. 2004. 151f. Dissertação (Mestrado em Geotecnia). Universidade de Brasília (ENC/FT/UnB), Setor de Geotecnia, Brasília-DF, 2004.

RICO, A.; DEL CASTILLO, H. La ingeniería de suelos en las vias terrestres. México, D.F.: Limusa, 1997. 643p.

SANT'ANA, A.P. et al. Influência do tempo decorrido entre mistura e compactação de misturas solo-escória de alto-forno moída ativada com cal. In: REUNIÃO ANUAL DE

PAVIMENTAÇÃO, 34., 2003, Campinas. Anais..., Campinas: 2003. p. 90-96. CD-ROM.

SENÇO, W. Manual de técnicas de pavimentação. São Paulo: Pini, 2001. 671p.

SILVA, D.P. Estudo do comportamento reológico de solos estabilizados com cimento: processos gerais de estabilização. 1968. 343f. Tese (Especialista, Laboratório Nacional de Engenharia Civil, Lisboa, Portugal, Proc. 52/8/3048). 1968. 